Pacific Journal of Mathematic 


\section{ON TWO-DIMENSIONAL CONVEX BODIES}

\section{A. C. Woods}

1. Introduction. Let $R_{n}$ denote Euclidean $n$-space. Select a cartesian coordinate system in $R_{n}$. Let $X_{1}, X_{2}, \cdots, X_{n}$ be $n$ linearly independent points of $R_{n}$ and with the usual point-vector notation we arrive at the set $\Lambda$ of all points

$$
g_{1} X_{1}+g_{2} X_{2}+\cdots+g_{n} X_{n}
$$

such that $g_{1}, g_{2}, \cdots, g_{n}$ are rational integers. The set $\Lambda$ is called a lattice and $X_{1}, X_{2}, \cdots, X_{n}$ are said to form a basis of the lattice $\Lambda$. For a given lattice the basis may be chosen in an infinite number of distinct ways. But if in coordinates $x_{i}=\left(x_{i 1}, x_{i 2}, \cdots, x_{i n}\right)$ for $i=1,2, \cdots, n$, then the absolute value of the determinant $\left\|x_{i j}\right\|$ is independent of the choice of basis. This number is called the determinant of the lattice $\Lambda$ and is denoted by $d(\Lambda)$.

A convex body $K$ of $n$ dimensions is a closed, bounded, convex set in $R_{n}$ with inner points. A lattice $A$ is said to be $K$-admissible if no point of $A$ other than the origin 0 is an inner point of $K$. The critical determinant $\Delta(K)$ of $K$ is then defined to be the infimum of $d(\Lambda)$ extended over all $K$-admissible lattices $\Lambda$.

Denote by $\mu_{1}(\Lambda), \mu_{2}(\Lambda), \cdots, \mu_{n}(\Lambda)$ the least upper bounds respectively of real numbers $c_{1}, c_{2}, \cdots, c_{n}$ such that $c_{i} K$ contains at most $i-1$ linearly independent points of $\Lambda$, for $i=1,2, \cdots, n$. The numbers $\mu_{1}(\Lambda), \mu_{2}(\Lambda)$, $\cdots, \mu_{n}(\Lambda)$ are called the successive minima of $\Lambda$ with respect to $K$. The question has been raised whether the inequality

$$
\mu_{1}(\Lambda) \mu_{2}(\Lambda) \cdots \mu_{n}(\Lambda) \Delta(K) \leqq d(\Lambda)
$$

is true for convex bodies $K$ that are symmetric in the origin 0 . This is known to hold for $n=2$ [1] and for $n=3$ [4] but the general case remains open. It is shown here that for $n=2$ the inequality (1) holds for convex bodies that are not necessarily symmetric in 0 . This result is then applied to extend to such bodies a theorem of Mahler's [2] on two-dimensional convex bodies symmetric in 0 .

2. Preliminary lemmas. Henceforth all considerations will be in $R_{2}$. Thus let $K$ be a two-dimensional convex body. The following lemmas are needed for the proofs of our theorems.

Lemma 1. Given a lattice $\Lambda$ there exists a lattice $\Lambda^{*}$ such that

Received February 10, 1958. 
(i ) $d\left(\Lambda^{*}\right)=d(\Lambda)$,

(ii) $\mu_{1}\left(\Lambda^{*}\right) \mu_{2}\left(\Lambda^{*}\right) \geqq \mu_{1}(\Lambda) \mu_{2}(\Lambda)$,

(iii) $\mu_{2}\left(\Lambda^{*}\right)<2 \mu_{1}\left(\Lambda^{*}\right)$.

A proof of this result has been given by Rankin [3] for $K$ symmetric in 0 . However his proof makes no use of the symmetry of $K$ and so we may refer the reader to it for a proof of the lemma. The next result is classical.

Lemma 2. If $X_{1}, X_{2}$ are two linearly independent points of a lattice $\Lambda$ such that the triangle with vertices $X_{1}, X_{2}, 0$ contains no point of $\Lambda$ apart from its vertices then $X_{1}, X_{2}$ form a basis of $\Lambda$.

Let $C$ be a convex set in $R_{2}$ and for any point $X$ in $R_{2}$ define the shadow $S(C, X)$ of $C$ in $X$ to be the set of points $Y$ such that the line segment $Y X$ produced past $X$ meets $C$. That is to say, $S(C, X)$ is the set of $Y$ such that $t X+(1-t) Y \in C$ for some $t>1$. Thus if $X$ is not an inner point of a convex body $K$ and $C \subset K$ then $S(C, X)$ does not contain an inner point of $K$. For assume that this assertion is false so that there is an inner point $Z$ say of $K$ which is also in $S(C, X)$. By definition of $S(C, X)$ the line segment $Z X$ produced past $X$ meets $C$ and therefore also $K$. This implies that $X$ is an inner point of $K$ contrary to the hypothesis.

LEMma 3. Let $K$ be a convex body containing the origin as an inner point. Let $X_{1}, X_{2}$ be a pair of linearly independent points of the boundary of $K$ such that no one of the points $-X_{1},-X_{2}, \pm X_{1}, \pm X_{2}$ is an inner point of $K$. Then the lattice generated by $X_{1}, X_{2}$ is $K$-admissible.

Proof. Take coordinates such that $X_{1}, X_{2}$ are the points $(1,0),(0,1)$ respectively. Let $C$ be the triangle with vertices $(0,0),(1,0),(0,1)$. Then $C \subset K$ from which it follows that no one of the sets $S\left(C, X_{1}\right)$, $S\left(C, X_{2}\right), S\left(C,-X_{1}\right), S\left(C,-X_{2}\right), S\left(C, X_{1}+X_{2}\right), S\left(C,-X_{1}+X_{2}\right), S\left(C, X_{1}-X_{2}\right)$, $S\left(C,-X_{1}-X_{2}\right)$ contains an inner point of $K$. But the union of these sets contains every point with integral coordinates other than 0 , that is to say it contains every point of the lattice generated by $X_{1}, X_{2}$ other than 0 . This completes the proof of the lemma.

3. On the successive minima. In this section we prove the following.

THEOREM 1. If $K$ is any convex body in $R_{2}$ and $\Lambda$ a lattice then

$$
\mu_{1}(\Lambda) \mu_{2}(\Lambda) \Delta(K) \leqq d(\Lambda) \text {. }
$$

Proof. If $K$ does not contain 0 as an inner point then $\Delta(K)=0$ and the theorem is trivial. We therefore assume from now on that 0 is an 
inner point of $K$. Now any convex body may be approximated arbitrarily closely by strictly convex bodies i.e. convex bodies such that their boundaries contain no line segment, so by an obvious continuity argument there is no loss of generality in assuming that $K$ is strictly convex. Finally by Lemma 1 it is evident that we may also assume $\mu_{2}(\Lambda)<2 \mu_{1}(\Lambda)$.

Let $X_{1}, X_{2}$ be two linearly independent points of $\Lambda$ such that $X_{1} \epsilon$ $\mu_{1}(\Lambda) K, X_{2} \in \mu_{2}(\Lambda) K$. It follows from the definition of successive minima and the strict convexity of $K$ that the triangle with vertices $0, X_{1}, X_{2}$ contains no point of $\Lambda$ apart from its vertices. By Lemma 2 , the points $X_{1}, X_{2}$ form a basis of $\Lambda$.

By definition of the successive minima no point of the form $g X_{1}$ where $g$ is a non-zero integer is in the interior of $\mu_{1}(A) K$ and no point of the form $f X_{1}+h X_{2}$ where $f, h$ are integers with $h \neq 0$ is in the interior of $\mu_{2}(A) K$.

Put $c=\mu_{1}(\Lambda)^{-1} \mu_{2}(\Lambda)$ so that $1 \leqq c<2$ and suppose that there exists a $\mu_{2}(\Lambda) K$-admissible lattice of determinant $c d(\Lambda)$. Then $\Delta\left(\mu_{2}(\Lambda) K\right) \leqq c d(\Lambda)$ or

$$
\mu_{1}(\Lambda) \mu_{2}(\Lambda) \Delta(K) \leqq d(\Lambda)
$$

and the theorem is true. It then remains to prove that there exists a $\mu_{2}(\Lambda) K$-admissible lattice of determinant $c d(\Lambda)$.

By a preceding remark no point of the form $g c X_{1}$ where $g$ is a nonzero integer is in the interior of $\mu_{2}(\Lambda) K$. Denote by $\Lambda^{*}$ the lattice generated by $c X_{1}, X_{2}$ of determinant $c d(\Lambda)$. If $\Lambda^{*}$ is $\mu_{2}(\Lambda) K$-admissible then the theorem is true. Hence we assume from now on that $A^{*}$ is not $\mu_{2}(\Lambda) K$-admissible. It is evident that $c X_{1}, X_{2}$ are boundary points of $\mu_{2}(A) K$. Applying Lemma 3 we see that one of the points $-c X_{1},-X_{2}$, $\pm c X_{1}, \pm X_{2}$ is an inner point of $\mu_{2}(\Lambda) K$. Clearly $-c X_{1},-X_{2}$ are not inner points of $\mu_{2}(A) K$. Moreover $c X_{1}+X_{2}$ is in the shadow $S\left(\left\{X_{2}\right\}, X_{1}+X_{2}\right)$ while $-c X_{1}+X_{2}$ is in the shadow $S\left(\left\{X_{2}\right\},-X_{1}+X_{2}\right)$ and as $X_{2} \in \mu_{2}(\Lambda) K$ whereas $X_{1}+X_{2},-X_{1}+X_{2}$ are not inner points of $\mu_{2}(\Lambda) K$ so also $c X_{1}+X_{2},-c X_{1}+X_{2}$ are not inner points of $\mu_{2}(\Lambda) K$. We conclude that either $c X_{1}-X_{2}$ or $-c X_{1}-X_{2}$ is an inner point of $\mu_{2}(\Lambda) K$. This is equivalent to saying that the line $t X_{1}-X_{2}$ with parameter $t$ meets $\mu_{2}(\Lambda) K$ in a line segment with endpoints $a_{1} X_{1}-X_{2}, a_{2} X_{1}-X_{2}$ such that

$$
\text { (i) } 1 \leqq\left|a_{1}\right|<c<\left|a_{2}\right| \leqq 2
$$

and

(ii) $a_{1}, a_{2}$ are of comparable sign.

On the other hand the line $t X_{1}+X_{2}$ with parameter $t$ meets $\mu_{2}(\Lambda) K$ in a line segment one endpoint of which is $X_{2}$ the other endpoint being of the form $b X_{1}+X_{2}$ where $0 \leqq|b| \leqq 1$. We distinguish the four cases arising when the sign of $a_{1}, a_{2}$ and the sign of $b$ are both taken into account. 


$$
a_{1}>0, \quad a_{2}>0 ; \quad b \geqq 0 .
$$

The lattice generated by the points $c X_{1}, a_{2} X_{1}-X_{2}$ is of determinant $c d(\Lambda)$. We assert that this lattice is $\mu_{2}(\Lambda) K$-admissible for assume that this is false. Since $c X_{1}, a_{2} X_{1}-X_{2}$ are both on the boundary of $\mu_{2}(\Lambda) K$ it follows from Lemma 2 that one of the points $-c X_{1},-a_{2} X_{1}+X_{2}$, $\left(c+a_{2}\right) X_{1}-X_{2},\left(c-a_{2}\right) X_{1}+X_{2}, \quad\left(a_{2}-c\right) X_{1}-X_{2},-\left(c+a_{2}\right) X_{1}+X_{2}$ is an inner point of $\mu_{2}(\Lambda) K$. But by what has been said already this is impossible, hence the lattice is $\mu_{2}(A) K$-admissible from which the theorem follows.

$$
a_{1}>0, \quad a_{2}>0, \quad b<0 .
$$

The lattice generated by the points $c X_{1}, a_{1} X_{1}-X_{2}$ is of determinant $c d(\Lambda)$. We assert that this lattice is $\mu_{2}(\Lambda) K$-admissible for assume that this is false. Since $c X_{1}, a_{1} X_{1}-X_{2}$ are both on the boundary of $\mu_{2}(\Lambda) K$ it follows from Lemma 2 that one of the points $-c X_{1},-a_{1} X_{1}+X_{2}$, $\left(c+a_{1}\right) X_{1}-X_{2}, \quad\left(c-a_{1}\right) X_{1}+X_{2}, \quad\left(a_{1}-c\right) X_{1}-X_{2},-\left(c+a_{1}\right) X_{1}+X_{2}$ is an inner point of $\mu_{2}(\Lambda) K$. But by what has already been said this is impossible, hence the lattice is $\mu_{2}(\Lambda) K$-admissible from which the theorem follows.

$$
a_{1}<0, \quad a_{2}<0 ; \quad b \geqq 0 .
$$

The lattice generated by the points $c X_{1}, a_{1} X_{1}-X_{2}$ is of determinant $c d(\Lambda)$. We assume that this lattice is $\mu_{2}(\Lambda) K$-admissible for assume that this is false. Since $c X_{1}, a_{1} X_{1}-X_{2}$ are both on the boundary of $\mu_{2}(\Lambda) K$ it follows from Lemma 2 that one of the points $-c X_{1},-a_{1} X_{1}+X_{2}$, $\left(c+a_{1}\right) X_{1}-X_{2}, \quad\left(c-a_{1}\right) X_{1}+X_{2}, \quad\left(a_{1}-c\right) X_{1}-X_{2},-\left(c+a_{1}\right) X_{1}+X_{2}$ is an inner point of $\mu_{2}(\Lambda) K$. But by what has been said already this is impossible, hence the lattice is $\mu_{2}(\Lambda) K$-admissible from which the theorem follows.

$$
a_{1}<0, \quad a_{2}<0 ; \quad b<0 \text {. }
$$

The lattice generated by the points $c X_{1}, a_{2} X_{1}-X_{2}$ is of determinant $c d(\Lambda)$. We assert that this lattice is $\mu_{2}(\Lambda) K$-admissible for assume that this is false. Since $c X_{1}, a_{2} X_{1}-X_{2}$ are both on the boundary of $\mu_{2}(\Lambda) K$ it follows from Lemma 2 that one of the points $-c X_{1},-a_{2} X_{1}+X_{2}$, $\left(c+a_{2}\right) X_{1}-X_{2}, \quad\left(c-a_{2}\right) X_{1}+X_{2}, \quad\left(a_{2}-c\right) X_{1}-X_{2},-\left(c+a_{2}\right) X_{1}+X_{2}$ is an inner point of $\mu_{2}(\Lambda) K$. But by what has already been said this is impossible, hence the lattice is $\mu_{2}(\Lambda) K$-admissible from which the theorem follows.

The above four cases exhaust all possibilities and so the theorem is proved.

4. A decreasing function. In this section we apply Theorem 1 to prove for any two-dimensional convex body a theorem of Mahler's [2] on two-dimensional symmetric convex bodies. Thus let $K$ again be any two-dimensional convex body and choose a coordinate system such that 
$\left(x_{1}, x_{2}\right)$ are the general coordinates of a point. Denote by $K(t)$ the set of points in $K$ that satisfy the inequality $\left|x_{2}\right| \leqq t$. Mahler [2] has shown that if $K$ is symmetric on the origin then $J(K(t)) / t$ is a decreasing function of $t$, for $t>0$. We will prove the following theorem.

THEOREM 2. If $K$ is any two-dimensional convex body then $\Delta(K(t)) / t$ is a decreasing function of $t$ for $t>0$.

Proof. If $K$ does not contain the origin as an inner point then $\Delta(K(t))=0$ for all $t>0$, and the theorem is trivial. So we assume from now on that 0 is an inner point of $K$. Further, appealing to a continuity argument similar to that employed in Theorem 1 it is evident that the truth of Theorem 2 for all strictly convex bodies $K$ implies its truth for any convex body. Hence we assume from now on that $K$ is strictly convex.

The theorem will be proved if for any given $t>0$ we can show $\Delta(K(s)) / s \leqq \Delta(K(t)) / t$ for all $s$ greater than $t$ and sufficiently close to $t$. Thus let $t>0$ be fixed. Denote by $\Lambda$ a critical lattice of $K(t)$, that is $\Lambda$ is a $K(t)$-admissible lattice such that $d(\Lambda)=\Delta(K(t))$. Let $N(\Lambda)$ be the number of points of $\Lambda$ which are on the boundary of $K(t)$ but which are not on the boundary of $K$, under one proviso, namely that if a pair of points of the form $\pm X$ both satisfy the above condition then the pair is to be counted as one point. We distinguish cases.

$$
N(\Lambda)=0 .
$$

Since any bounded region of the plane contains only a finite number of points of $\Lambda$ it follows that there exists an $\varepsilon>0$ such that $\Lambda$ is $K(t+\varepsilon)$-admissible and therefore also $K(s)$-admissible provided only that $t \leqq s \leqq t+\varepsilon$. But for such values of $s, K(t) \subset K(s)$ and thus $\Delta(K(t))=$ $\Delta(K(s))$ whence

$$
\Delta(K(t)) / t \geqq \Delta(K(s)) / s .
$$

$$
N(\Lambda)=1 .
$$

Denote by $X$ a point of $\Lambda$ on the boundary of $K(t)$ but not on the boundary of $K$. If there is another such point then it is necessarily- $X$. There exists an $\varepsilon>0$ such that the ray $0 X$ produced meets the boundary of $K(t+\varepsilon)$ in an inner point of $K$ and such that $K(t+\varepsilon)$ contains no point of $\Lambda$ within its interior other then 0 and $\pm X$. Let $s$ be such that $t \leqq s \leqq t+\varepsilon$ so that $K(s)$ also contains no point of $\Lambda$ within its interior apart from 0 and $\pm X$. Let further $\mu_{1}(\Lambda), \mu_{2}(\Lambda)$ denote the successive minima of $\Lambda$ with respect to $K(s)$. Evidently $\mu_{2}(\Lambda) \geqq 1$ and moreover $X$ lies on the boundary of $\mu_{1}(\Lambda) K(s)$. But $X$ lies on the boundary 
of $K(t)$ and furthermore the ray $0 X$ produced meets the boundary of $K(s)$ in an inner point of $K$. Therefore $\mu_{1}(\Lambda)=t / s$. By Theorem 1

hence

$$
\mu_{1}(\Lambda) \mu_{2}(\Lambda) \Delta(K(s)) \leqq d(\Lambda)
$$

$$
(t / s) \Delta(K(s)) \leqq \Delta(K(t))
$$

or

$$
\Delta(K(s)) / s \leqq \Delta(K(t)) / t
$$$$
N(\Lambda) \geqq 2 \text {. }
$$

There exist two linearly independent points $X_{1}, X_{2}$ say of $\Lambda$ on the boundary of $K(t)$ but not on the boundary of $K$. This implies that their $x_{2}$-coordinates both satisfy the equality $\left|x_{2}\right|=t$. If their $x_{2}$-coordinates both have the same value we may assume $X_{1}, X_{2}$ to be chosen so that the line segment connecting the two points contains no further point of $\Lambda$. It then follows from the strict convexity of $K$ and the fact that 0 is an inner point of $K$ that the triangle with vertices $0 X_{1} X_{2}$ contains no point of $\Lambda$ apart from its vertices. By Lemma 2 the vectors $X_{1}, X_{2}$ form a basis of $\Lambda$. But either $X_{1}, X_{2}$ both lie on the line $x_{2}=t$, or one lies on this line while the other lies on the line $x_{2}=-t$, or else they both lie on the latter line. In all cases the points of $\Lambda$ are confined to the lines $x_{2}=n t, n=0, \pm 1, \pm 2, \cdots$. For given $s \geqq t$ denote by $\Lambda(s)$ the set of points $\left(x_{1}, s x_{2} / t\right)$ where $\left(x_{1}, x_{2}\right) \in \Lambda$. Then $\Lambda(s)$ is a lattice of determinant $d(\Lambda(s))=(s / t) d(\Lambda)=(s / t) \Delta(K(t))$. Moreover $\Lambda(s)$ is $K(s)$-admissible since all points of $\Lambda(s)$ lie on the lines $x_{2}=n s, n=0, \pm 1, \pm 2, \cdots$ and those that lie on the line $x_{2}=0$ coincide with those points of $\Lambda$ lying on this line and these points other than 0 are not inner points of $K$ so also are not inner points of $K(s)$. Hence

$$
\Delta(K(s)) \leqq d(\Lambda(s))=(s / t) \Delta(K(t))
$$

or

$$
\Delta(K(s)) / s \leqq \Delta(K(t)) / t
$$

The above three cases exhaust all the possibilities and we conclude that the theorem is true.

Part of this work was done at the University of Manchester under the supervision of Professor Kurt Mahler to whom I am very grateful for advice and encouragement at all times.

\section{REFERENCES}

1. C. Chabauty, Ann. Sci. Ec. Norm. Sup., Paris, (3) 66 (1949), 367-394.

2. K. Mahler, Rend. Mat. Appl., Serie V, 13 (1954).

3. R. A. Rankin, Proc. Cambridge, Philos. Soc. 49 (1953), 54-58.

4. A. C. Woods, Proc. Cambridge Philos. Soc. 52 (1956), 406-423. 


\section{PACIFIC JOURNAL OF MATHEMATICS}

\section{EDITORS}

David Gilbarg

Stanford University

Stanford, California

R. A. Beaumont

University of Washington

Seattle 5, Washington

\author{
A. L. Whiteman
}

University of Southern California

Los Angeles 7, California

E. G. Straus

University of California

Los Angeles 24, California

\section{ASSOCIATE EDITORS}

\author{
E. F. BECKENBACH \\ C. E. BURGESS \\ M. HALL \\ E. HEWITT
}

\author{
A. HORN \\ V. GANAPATHY IYER \\ R. D. JAMES \\ M. S. KNEBELMAN
}

L. NACHBIN
I. NIVEN
T. G. OSTROM
H. L. ROYDEN

M. M. SCHIFFER

G. SZEKERES

F. WOLF

K. YOSIDA

\section{SUPPORTING INSTITUTIONS}

\author{
UNIVERSITY OF BRITISH COLUMBIA \\ CALIFORNIA INSTITUTE OF TECHNOLOGY \\ UNIVERSITY OF CALIFORNIA \\ MONTANA STATE UNIVERSITY \\ UNIVERSITY OF NEVADA \\ OREGON STATE COLLEGE \\ UNIVERSITY OF OREGON \\ UNIVERSITY OF SOUTHERN CALIFORNIA
}

\author{
STANFORD UNIVERSITY \\ UNIVERSITY OF UTAH \\ WASHINGTON STATE COLLEGE \\ UNIVERSITY OF WASHINGTON \\ AMERICAN MATHEMATICAL SOCIETY \\ CALIFORNIA RESEARCH CORPORATION \\ HUGHES AIRCRAFT COMPANY \\ THE RAMO-WOOLDRIDGE CORPORATION
}

Mathematical papers intended for publication in the Pacific Journal of Mathematics should be typewritten (double spaced), and the author should keep a complete copy. Manuscripts may be sent to any of the editors. All other communications to the editors should be addressed to the managing editor, E. G. Straus at the University of California, Los Angeles 24, California.

50 reprints per author of each article are furnished free of charge; additional copies may be obtained at cost in multiples of 50 .

The Pacific Journal of Mathematics is published quarterly, in March, June, September, and December. The price per volume (4 numbers) is $\$ 12.00$; single issues, $\$ 3.50$. Back numbers are available. Special price to individual faculty members of supporting institutions and to individual members of the American Mathematical Society: $\$ 4.00$ per volume; single issues, $\$ 1.25$.

Subscriptions, orders for back numbers, and changes of address should be sent to Pacific Journal of Mathematics, 2120 Oxford Street, Berkeley 4, California.

Printed at Kokusai Bunken Insatsusha (International Academic Printing Co., Ltd.), No. 6, 2-chome, Fujimi-cho, Chiyoda-ku, Tokyo, Japan.

\section{PUBLISHED BY PACIFIC JOURNAL OF MATHEMATICS, A NON-PROFIT CORPORATION}

The Supporting Institutions listed above contribute to the cost of publication of this Journal, but they are not owners or publishers and have no responsibility for its content or policies. 


\section{Pacific Journal of Mathematics}

\section{Vol. 8, No. 3 \\ May, 1958}

Michael Israel Aissen, A set function defined for convex plane domaines... . 383

Robert Ellis, Distal transformation groups ................... 401

Ciprian Foias, On a commutative extension of a commutative Banach algebra ....................................... 407

Jerry William Gaddum, Linear inequalities and quadratic forms ......... 411

Allen A. Goldstein and Elliott Ward Cheney, Jr., A finite algorithm for the solution of consistent linear equations and inequalities and for the Tchebycheff approximation of inconsistent linear equations...........

William L. Hart and T. S. Motzkin, Proof of the fundamental theorem on implicit functions by use of composite gradient corrections .......... 429

Henry Berge Helson, Conjugate series and a theorem of Paley .......... 437

Wu-Chung Hsiang, Abelian groups characterized by their independent subsets....................................... 447

John W. Lamperti, On the isometries of certain function-spaces ........ 459

Karel DeLeeuw and Walter Rudin, Extreme points and extremum problems

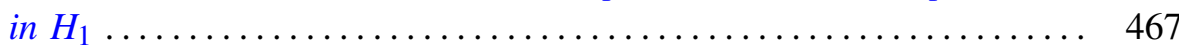

Eugene Lukacs, Some extensions of a theorem of Marcinkiewicz........ 487

George W. Mackey, Multiplicity free representations of finite groups ..... 503

Eben Matlis, Injective modules over Noetherian rings ............. 511

John William Neuberger, Continuous products and nonlinear integral equations

Lawrence Edward Payne and Hans F. Weinberger, New bounds for solutions of second order elliptic partial differential equations...

William T. Reid, A Prüfer transformation for differential systems ........ 575

Howard L. Rolf, The free lattice generated by a set of chains ...

K. M. Saksena, Inversion and representation theorems for a generalized

Laplace integral....................................... 597

Daniel Shanks, Two theorems of Gauss......................... 609

Paul Slepian, On the Lebesgue area of a doubled map ............... 613

Otto Szász and Nelson Paul Yeardley, Jr., The representation of an analytic function by general Laguerre series . ..................... 621

Alan C. Woods, On two-dimensional convex bodies ................. 635 